Ilaria Gabbatore*, Francesca M. Bosco, Leena Mäkinen, Eeva Leinonen and Soile Loukusa

\title{
Social-pragmatic contextual comprehension in Italian preschool and school-aged children: a study using the Pragma test
}

https://doi.org/10.1515/ip-2021-2001

\begin{abstract}
Efficient communication requires the interplay of linguistic, cognitive and social skills, including the ability to make contextual inferences and to understand others' intentions and emotions. The capacity to effectively use language in specific contexts (i.e., pragmatic ability) develops with age, and an assessment of this ability is important for understanding both typical and atypical development. The Pragma test was originally developed and validated on Finnish children to assess social-pragmatic comprehension. The present study utilizes a slightly adapted version of the Pragma test, translated into Italian, and presents the results of the test given to 110 typically developing Italian children (4-8 years of age). The Italian version of the Pragma test shows content and concurrent validity, interrater reliability, and internal consistency, and it proves to be sensitive in detecting an age-dependent pattern of performance, across pre-school and school age children, in the pragmatic parameters investigated. The present study has ramifications for clinical contexts, as data for typical pragmatic development enables identification of strengths and weaknesses in the pragmatic performance in clinical populations. Finally, the results indicate that the Pragma test is relevant to both Finnish and Italian cultural contexts, thus providing the opportunity to make cross-cultural comparisons.
\end{abstract}

Keywords: contextual inference, language comprehension, Pragma test, pragmatic ability, theory of mind

\section{Introduction}

Children take part daily in many different types of communicative interactions with peers and adults, at home and at school. Being able to communicate in an

*Corresponding author: Ilaria Gabbatore, University of Turin, Turin, Italy,

E-mail: ilaria.gabbatore@unito.it

Francesca M. Bosco, University of Turin, Turin, Italy

Leena Mäkinen and Soile Loukusa, University of Oulu, Oulu, Finland

Eeva Leinonen, Murdoch University, Perth, Australia

¿ Open Access. ( 2021 llaria Gabbatore et al., published by De Gruyter. ((c))BY under the Creative Commons Attribution 4.0 International License. 
effective way requires the interplay of linguistic, cognitive and social skills, including the ability to direct attention towards relevant contextual elements as well as infer and understand others' intentions, aims and emotions (Cummings 2009; Perkins 2007; Sperber and Wilson 2012).

Pragmatic ability refers to the capacity to effectively use language in specific contexts (Levinson 1983) and enables a person to infer and utilize relevant contextual information in utterance interpretation and expression, in different kinds of communication situations (Sperber and Wilson 1995). The ability to make inference enables one to connect and link different kinds of information (Leinonen et al. 2000; Sperber and Wilson 2012), thus playing an important role in 'filling the gaps' between the literal and the intended meaning of an utterance (Searle 1975). Such skill develops with age (Angeleri and Airenti 2014; Bosco and Gabbatore 2017; Loukusa and Ryder 2007; Loukusa et al. 2008; Ryder and Leinonen 2003; see also Grigoroglou and Papafragou 2017; Matthews 2014). Acceleration of this development is particularly evident between the ages of four and eight, when children's ability to utilize contextually relevant information (Bosco et al. 2004; Loukusa and Leinonen 2008) and utilize different kinds of contextual information in language comprehension (Loukusa et al. 2007) increases significantly. Children develop the ability to go beyond the propositional meaning of a sentence, when interpreting a communicative situation.

Similarly, Theory of Mind (ToM) - i.e., the ability to understand one's own and others' mental states and behave accordingly (Premack and Woodruff 1978) develops through childhood (Saxe 2013; Wellman and Liu 2004) and continues into adolescence (Bosco et al. 2014, 2016; Brizio et al. 2015). ToM appears to play a role when it comes to pragmatic comprehension as, for example, the ability to understand others' mental states seems to correlate with the ability to understand lies and deceits (Cheung et al. 2015; Sodian and Frith 1992), ironies (Nilsen et al. 2011; Winner and Leekam 1991; Sullivan et al. 1995), communicative failures (Bosco and Gabbatore 2016), and mental metaphors (Lecce et al. 2019). Matthews et al. (2018) recently analyzed the relationship between pragmatic language and mentalizing ability, considering also the role of formal language and other cognitive functions, in typical and atypical development. The authors highlighted that mentalizing was able to explain only some of the variance in the pragmatic skills, i.e., discourse contingency and comprehension of irony. On the other end, in a very recent study, Cardillo and colleagues (2020) assessed pragmatic language in children and adolescents with Autism Spectrum Disorder, finding a link between nonliteral language, the capacity to make inferences and ToM skills. Despite the evidence regarding a link between these abilities, the nature of the relationship between pragmatic and ToM abilities is still not well understood (Bosco and Gabbatore 2017; Bosco et al. 2018; Maurizio and Bosco 2008). This may be partly due to different theoretical approaches that have 
been adopted to look at such complex and interconnected human abilities: this is, for example, the case when we consider the difficulty of integrating the concept of Theory Mind, arising from experimental studies in cognitive psychology, with the notion of intersubjectivity, adopted in usage-based linguistics, reflecting similar but differently examined Aspects of social cognition (see Tantucci 2020 and Tantucci and Wang 2020 for a comprehensive cross-disciplinary analysis of this topic).

In addition to the ability to understand contextual and social meanings in communicative situations, also being able to reflect on and explain one's own comprehension process, is an ability that develops with age. Explaining one's answers is a verbal activity, demanding many cognitive abilities, such as recognizing the difference between action and intention (Donaldson 2006). The ability to give relevant explanations to answers that are demanding from the point of view of contextual processing is challenging since they require, for example, the ability to distinguish between cause and effect as well as the ability to manipulate premises required in deriving a conclusion. Current research suggests that becoming fully aware of one's own processing in different situations and being able to give a verbal explanation on request develops over a period of time (Donaldson 2006; Letts and Leinonen 2001; Loukusa et al. 2008, 2017). Donaldson (2006) highlighted that although three-year-old children were able to distinguish between cause and effect, it is not until the age of eight that children are able to use "because" or "so" when giving deductive explanations as evidence supporting a conclusion. This finding was later supported by Loukusa et al. (2008, 2017). Although, in Loukusa et al. (2017) study, five-year-old children managed to explain many of their correct answers, it was only at the age of eight when they reached $80 \%$ for correct explanations. As children develop they become more able to explicitly express the information they had used in arriving at their answers. In this regard, literature focuses on the relationship between knowledge and belief representations, and on the distinction between the ability to identify behavioral cues of others' actions and the ability to discern others' beliefs and needs. A fine-grained analysis of these components is out of the scope of the present paper. Nevertheless, it is worth bearing in mind that these capacities show different developmental patterns and different degree of impairment in clinical population (see Apperly 2010; Phillips et al. 2020 for a wider discussion), and these are relevant to the study of pragmatic development.

Investigating the typical pattern of pragmatic development helps professionals to evaluate and support children's well-being and intervene whenever needed. Having difficulty in pragmatic processing can lead to problems in relationship formation (e.g., Whitehouse et al. 2009), as well as behavioral, social, and emotional difficulties (e.g., St Clair et al. 2011). During pre-school and school years, children utilize their social-pragmatic abilities in making friends (Gertner et al. 1994) as well 
as in interacting with teachers (Edwards and Mercer 1986). Miscommunication can result in social difficulties, including discrimination among peers (see ContiRamsden and Botting 2004; Mackie and Law 2010). It is for these kinds of reasons why pragmatic processing difficulties should be given consideration, and supported whenever needed, from an early age, throughout school years and into adolescence. Also, within this picture, it is also relevant to consider children's socio economical background, that has been shown to impact on many aspects of child development, particularly language development (e.g., Hoff-Ginsberg 1998; Row 2008). Nevertheless, studies targeting specifically pragmatic abilities in school aged children failed to detect a specific role of socio-economic background on the observed performance, at least in the Italian context (e.g., Bosco and Gabbatore 2017; Bosco et al. 2013), thus suggesting that pragmatic abilities might be only partially affected by socioeconomic background but are influenced more clearly by other factors.

Finally, it is important to understand typical development of pragmatic abilities in order to be able to detect difficulties and delays in atypical development (i.e., clinical populations). A large body of literature highlights that social-pragmatic difficulties are core characteristics in many developmental disorders, as in Autism Spectrum Disorders (ASD; e.g., Angeleri et al. 2016; Loukusa and Moilanen 2009), Attention Deficit Hyperactivity Disorders (ADHD; e.g. Loukusa 2017a; Väisänen et al. 2014), developmental language disorder (DLD; e.g. Conti-Ramsden and Botting 2004; Letts and Leinonen 2001; Ryder et al. 2008), as well as in hearing impairment (e.g., O’Reilly et al. 2014).

Studies of the development of pragmatic and social skills in typical and atypical populations have created a variety of tasks and tools for the assessment of various aspects of pragmatic ability. The Test of Pragmatic Language (TOPL-2; Phelps-Terasaki and Phelps-Gunn 2007) is focused on children's (6-12 years of age) ability to generate pragmatically appropriate responses to presented scenarios and specifically focuses on sub-components of pragmatics like physical setting, audience, topic, purpose, gestural cues, and abstraction. The Clinical Evaluation of Language Fundamentals $^{\circledR}$, Fifth Edition Metalinguistics $\left(\right.$ CELF $^{\circledR}-5$ Metalinguistics; Wiig and Secord 2014), suitable for age range of 9-21, focuses on the ability to understand ambiguous sentences and on listening comprehension, on making inferences, on recreating speech acts and on figurative language. The Targeted Observation of Pragmatics in Children's Conversations (TOPICC; Adams et al. 2011) assesses components such as taking into account the listener knowledge, turn-taking, verbosity, topic management, discourse style and problem solving. The Assessment Battery for Communication (ABaCo; Angeleri et al. 2012) and its equivalent forms (Bosco et al. 2012) include a wide range of phenomena, looking at both comprehension and production, and through different expressive modalities. ABaCo has initially been used with adults with brain injuries and psychiatric conditions 
(Angeleri et al. 2008; Colle et al. 2013; Gabbatore et al. 2014), and a version has been adapted for children. This has been shown to be sensitive in detecting developmental patterns in typically developing children (Bosco et al. 2013) and in adolescents with ASD (Angeleri et al. 2016). Finally, the Pragma test (Loukusa 2019), described later in the present paper, focuses on children's social-pragmatic comprehension and has been successfully utilized in understanding typical pragmatic development among Finnish children (Loukusa et al. 2017), in Loukusa et al. (2018), among children with Specific Language Impairment (Loukusa et al. 2019), and children and young adults with ADHD (Loukusa 2017b).

There are also assessment protocols that are based on parent/teacher reports. In these kinds of assessments there is an advantage in that communicative behaviors are observed in social contexts and not only in a controlled test situation. However, these observation protocols have the limitation of the subjectivity of the observer. This is the case of the widely used Children's Communication Checklist (CCC-2; Bishop 2003), developed to screen for potential communication disorders and subclassify children and adolescents ranging from 4-16 years of age. The CCC-2 assesses speech, vocabulary, sentence structure and social language skills, and includes some specific measure for pragmatic ability, focusing on initiation, stereotyped language, use of context and non-verbal communication.

There is a variety of different measures, developed worldwide, to assess pragmatic ability from different perspectives. This results in challenges for being able to compare results of studies that use these different methodologies. It is also worth noting that most of the protocols have been developed in English and adapted to the Italian (e.g., CCC-2) and only a few tools have been developed in the Italian context (e.g. ABaCo). The Pragma test was developed and validated in Finnish (Loukusa 2019) taking into consideration previous data from the literature and it has been shown to enable the investigation of a wide range of social-pragmatic abilities in younger (starting from four years of age) children (see Loukusa et al. 2017). The Pragma test focuses on a person's ability to utilize contextual cues and understand intentions in pragmatic comprehension, areas that are not fully covered by other protocols (see also O'Neill 2012, 2014). The Pragma test has also been shown to be developmentally sensitive to detect social-pragmatic inference difficulties in ADHD (Loukusa 2017b), as well as in ASD (Loukusa et al. 2018), which makes it a valuable tool, with properties that enable us to integrate existing literature and outcomes in the clinical practice. The advantage of the Pragma test in comparison to a screening questionnaire, as in the case of the CCC-2, is the assessment protocol that is not mediated by caregiver observations, thus leading to more structured and quantifiable results. Given this, the Pragma test enables the detection of strengths and weaknesses in the development of social-pragmatic abilities. Moreover, the impact of cultural features on the communicative style of each country has been reported by 
a few studies (e.g., Küntay et al. 2014), suggesting that some aspects of communicative competence could be culture- or language-sensitive. Translating and adapting assessment protocols into different languages have a number of advantages, including cross-cultural comparisons, but this also poses challenges (see Bornman et al. 2010). While a few studies have been conducted regarding narrative skills (e.g., Colletta et al. 2014; Huttunen et al. 2013; Mäkinen et al. 2020), the development of pragmatic abilities across different languages and cultures has been investigated less (see Gabbatore et al. 2019), mainly because of lack of appropriate assessment protocols.

Finally, a further and not so investigated area of interest concerns the role of sex on pragmatic ability. While some studies have indicated differences regarding linguistic skills, with girls learning vocabulary faster (Roulstone et al. 2002), using language earlier (Murray et al. 1990) and exhibiting more spontaneous conversations (Bauer et al. 2002), to the best of our knowledge no specific data are available regarding sex differences in terms of pragmatic ability.

\section{Aims of the present study}

This study has two main aims:

1. To provide data regarding the psychometric properties of the translation and adaptation into Italian of the Pragma test, originally developed in Finnish (Loukusa et al. 2017, 2019), showing its validity and sensitivity.

2. To investigate how Italian children in 4-8 age groups differ in their ability to answer different kinds of social-pragmatic tasks, and specifically to verify the sensitivity of the Pragma test to detect - in its Italian version - a specific pattern of performance at different ages (four to eight years) in the social-pragmatic abilities investigated; moreover, we aim to explore whether any difference in the pragmatic abilities investigated may be detected between boys and girls.

The items composing the Pragma test are based on simple scenarios, which make the tool suitable for pre-school and school age children, with a focus on specific aspects of social-pragmatic comprehension. Preschool and school-age are important developmental periods for a number of pragmatic, cognitive and social skills, and typical data for these age groups will be informative for future studies with children with clinical conditions.

The Pragma test investigates children's performance on different types of tasks, namely: (a) Contextual inference - not implying ToM, (b) Contextual inference implying ToM, (c) Relevant language use, depending on the communicative context, (d) Emotion recognition, and (e) Understanding of False Beliefs. We expect to detect 
an increase in children's pragmatic performance with age, in line with previous studies (e.g., Angeleri and Airenti 2014; Bosco et al. 2013; Loukusa et al. 2007, 2008, 2017). Moreover, as the items composing the Pragma test suggest different underlying abilities, we expect to detect differences in the children's performance in the different types of tasks. In particular, we expect to observe that the questions requiring Theory of Mind abilities will be more demanding, in line with previous study of Finnish children (Loukusa et al. 2007, 2017). These types of questions, indeed, not only require drawing conclusions based on information derived from different sources, but also by taking into consideration another person's mental states, i.e. theory of mind. As ToM abilities increase in childhood (e.g., Wellman and Watson 2001; Wellman and Liu 2004), and an increase in such abilities is still observable during adolescence (e.g., Bosco et al. 2014), we expect the children in this study will not have fully mastered such skills and will have a high degree of variability depending on the specific task and age.

We also aim to explore the children's metacognitive abilities, that are reflected in their awareness of the reasoning that led them to a correct answer. A number of the Pragma test items specifically focus on metacognition and we expect to observe a different performance depending on the children's age, in line with earlier studies of Finnish children (Letts and Leinonen 2001; Loukusa et al. 2008, 2017), which have suggested this ability increases significantly between the age of four and eight.

\section{Method}

\subsection{The Pragma test: structure and coding}

The Pragma test (Loukusa 2019, see also Loukusa et al. 2017, 2018), originally developed in Finnish, is designed for pre-school and school aged children and consists of a set of tasks focused on the assessment of the ability to comprehend contextual meanings, intentions and relevant language use. The questions of the Pragma test were developed in order to try to systemize and distinguish between these different facets of pragmatic abilities. The tasks in the Pragma test consist of short scenarios, presented verbally to children with the support of colored pictures and plastic characters in order to minimize memory load.

The Test includes 39 questions, which are classified based on earlier literature in ASD and pragmatics (see e.g. Loukusa and Moilanen 2009). Correct answers require the ability to understand the implied meaning of the utterances in the scenarios. Questions vary according to contextual utilization, social language use and understanding of intentions, thoughts, beliefs and feelings. While being 
conscious of the multidimensional aspects of the processing demands of the questions, the questions are classified into categories according to the phenomenon that each question is primarily focusing on (Table 1).

In the Pragma test, the questions vary in number. Most of the questions measure contextual inference with ToM because it has been shown that particularly these kinds of questions cause difficulty for children with social-pragmatic difficulties, such as ASD (see e.g. Loukusa and Moilanen 2009). Since the ability to derive a conclusion by connecting information from different sources is central to pragmatic understanding (Leinonen et al. 2000; Sperber and Wilson 1995), the second most common question type is Contextual inference without ToM demand.

In addition, 13 of the questions also require the children to provide an explanation for their correct answers (How do you know that?). These explanation tasks are aimed at exploring whether and to what extent the children are aware of the way they used contextual information to derive the correct answer.

Each child is seen individually to take part in the test and the session is videorecorded for scoring. Each question is assigned a score of one if the child's answer displays an accurate comprehension of the communicative situation and addresses the pragmatic aspect investigated; if there is no evidence of this, the score of 0 is assigned to the answer. If a child answers a question correctly, a follow up question - explanation - is asked which asks the child to explain her/his answer. The purpose of this is to investigate whether the children are aware of and hence able to explain explicitly what factors (verbal and pictorial context, world and social knowledge) led them to the correct answer, giving some insight into their comprehension processes.

\subsection{Translation and adaptation of the Pragma test in Italian}

The Pragma test was originally designed in Finnish and then an English version was developed. For the purposes of this study, the test was translated into Italian from English and then back-translated into English in order to test its faithfulness to the original. Two experts in pragmatics (a Finnish and an Italian researcher) performed a cross check, directly comparing the Italian and the Finnish items, in order to verify the translation and assure that the content, meaning and structure of the Italian version corresponded to the original one. The items were then evaluated by three Italian graduate students of psychology, blind with respect to the aims and the original structure of the test, and later by two Italian researchers, in order to make sure that each item was relevant and authentic in the Italian version. Through this process very few modifications were required for the Italian version of the Pragma test. Some adjustments had to be made because of different cultural contexts. For 


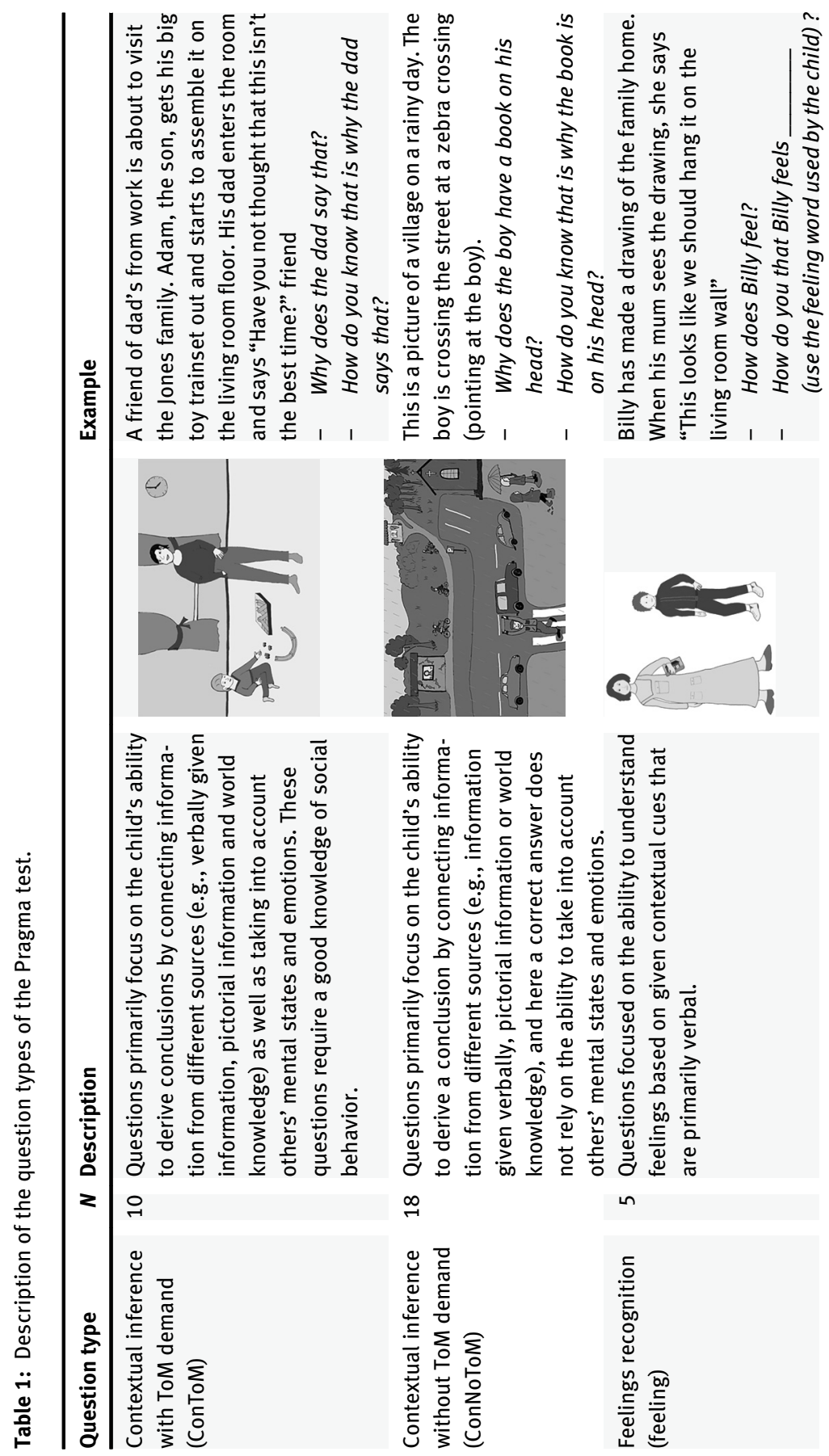




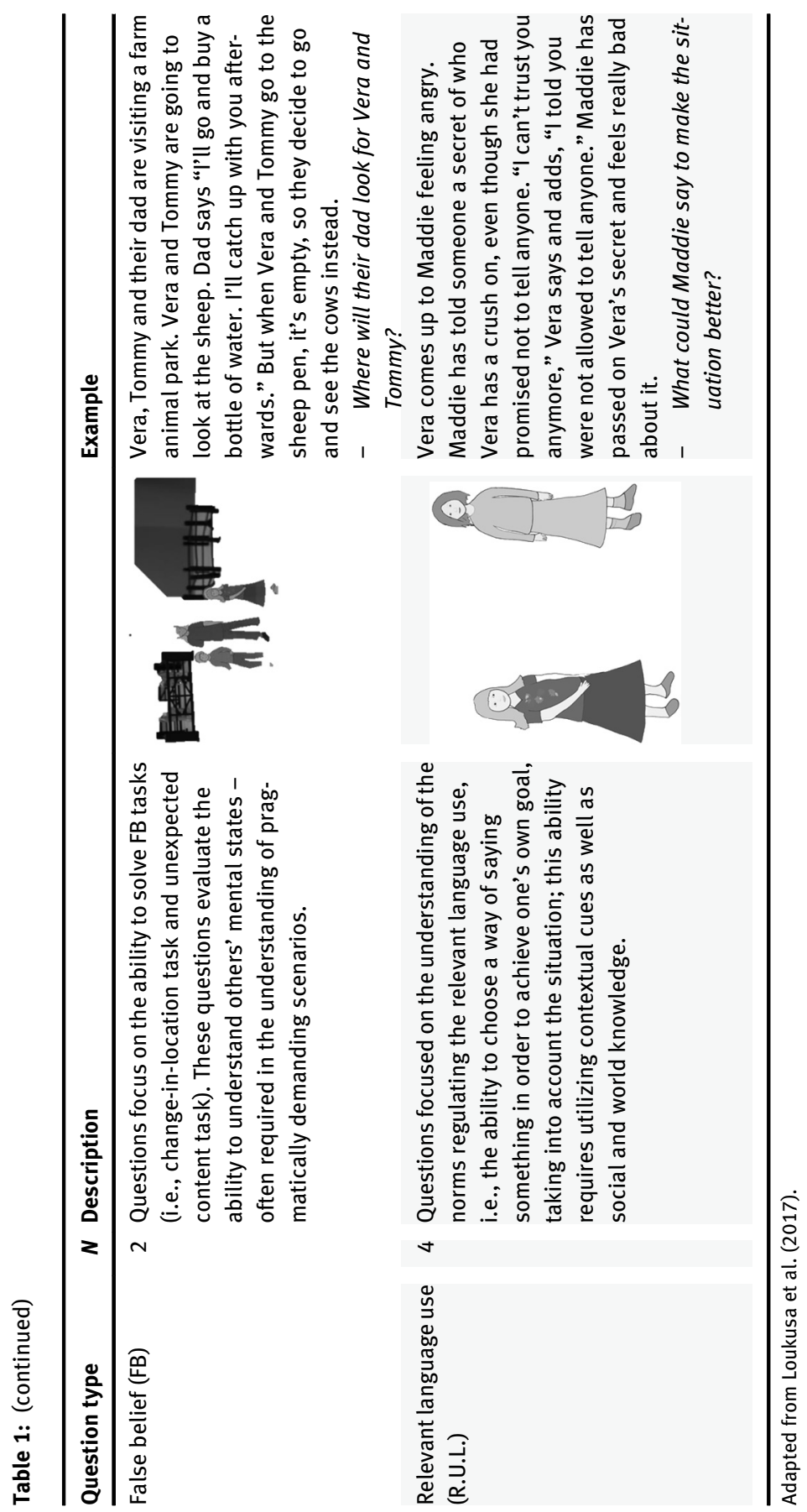


example, in item 31 (ConToM), in the Finnish version the child gets a sledge as a present for his birthday; in the Italian version, the sledge was substituted with a bike, which is more familiar to Italian children. Similarly, in item 13 (ConNoToM), the child is asked to observe a picture of a village on a rainy day. A small modification was made into the picture where the Finnish word kioski (kiosk) was removed from one of the depicted buildings. No other modifications were required, as the rest of the material was relevant and understandable for Italian children. The final version was then approved by the Finnish developers of the original test.

\subsection{Content validity}

A group of 40 Italian young adults (graduate students at the University of Turin) were then recruited in order to get a measure of content validity from a broader perspective. For each item, the volunteers were presented with the test stimulus (verbal scenario and picture/characters) and the test question: they were given a written form comprising a set of statements where the capability of the test item to measure the target domain was claimed (e.g., for question type Contextual inference without ToM demand, the statement was: "This item investigates the ability to make contextual inferences not requiring theory of mind") and they were then asked to rate the statement on a five-point Likert-type scale (disagree; slightly disagree; neither agree nor disagree; slightly agree; agree). Scoring was computed by attributing one to five points for each item (from 1 for disagree to 5 for agree). For each item, the mean of the values was $\geq 4$, with the only exception of two items reporting a mean value slightly <4. Looking at the evaluation scores assigned to the items grouped by scales: ConNoToM: mean value $3.97(\mathrm{SD}=0.48)$, ConToM: mean value 4.45 $(\mathrm{SD}=0.37)$, Feeling: mean value $4.69(\mathrm{SD}=0.35), F B$ : mean value $4.80(\mathrm{SD}=0.40)$, and $R U L$ : mean value $4.43(\mathrm{SD}=0.45)$. Overall, this showed that the items were appropriate to the Italian context.

\subsection{Interrater reliability and internal consistency}

In order to check the reliability of scoring, interrater reliability (intra-class correlation coefficient) was calculated between the scores assigned by two independent coders. The intra-class correlation coefficient between the two raters was calculated on a subsample of 11 children ( $10 \%$ of the total sample): the intra-class correlation coefficient was 0.985 for the scoring of the answers, and 0.984 for the scoring of the explanations, indicating that the scoring was highly reliable (Shrout 1998).

Cronbach's alpha showed excellent internal consistency of the battery in answers $(\alpha=0.927)$ and good internal consistency in explanations $(\alpha=0.794)$. 


\subsection{Concurrent validity}

In addition to the Pragma test, we also administered the Italian version (Di Sano et al. 2013) of the Children's Communication Checklist - II Edition (CCC-2; Bishop 2003), a questionnaire completed by a caregiver which enables an observation based screening for communication difficulties and the identification of pragmatic/social interaction problems (Norbury et al. 2004). The CCC- 2 is composed by 70 items, organized in 10 scales: (a) Speech, (b) Syntax, (c) Semantics, (d) Coherence, (e) Inappropriate initiation, (f) Stereotyped language, (g) Use of context, (h) Non-verbal communication, (i) Social relationships and (j) Interests. In each scale five items describe problematic behaviors (weaknesses) and two items describe positive behaviors (strengths); the caregiver is required to evaluate how frequently the behaviors occur. The CCC-2 is widely used in exploring children's pragmatic functioning and therefore we chose to include this in the present study in order to derive a measure of concurrent validity for the children's performance on the Pragma test.

A first correlation was run with the General Communication Composite (GCC) score, that is a value based on the scores - corrected for age - obtained on the first eight scales (Speech, Syntax, Semantics, Coherence, Inappropriate initiation, Stereotyped language, Use of context, Non-verbal communication) and designed to discriminate between children with communication impairments from typically developing children. A Pearson's correlation coefficient between the Pragma overall score and the General Communication Composite score of CCC-2 $(M=85.10$; $\mathrm{SD}=15.86)$ revealed a significant but a small correlation $(r=0.215 ; p=0.025)$. Moreover, in order to provide a more detailed overview, we performed a correlation analysis (Pearson $r$ ) between the total raw score of the Pragma test in the overall sample and children's raw scores at all the scales of the CCC-2 (see Table 2). Negative correlation in almost all the scales, with the exception of scale (i) social relation, suggest that, as expected, a higher score in the Pragma test corresponds to fewer communicative difficulties, as detected by the CCC-2.

\subsection{Administration of Pragma to the Italian sample}

\subsubsection{Participants}

The Pragma test was administered to a sample of 110 Italian typically developing children (56 girls, 54 boys). They were ranging in age from four and eight years $(M=6 ; 5, \mathrm{SD}=1 ; 6)$ and they were allocated into subgroups as detailed in Table 2. 
Table 2. Mean values (standard deviation) of the raw scores obtained at the scales of the CCC-2, and correlation (Pearson $r$ ) with the total score of the Pragma test.

\begin{tabular}{lrr}
\hline CCC-2 Scale & $\boldsymbol{M}(\mathrm{SD})$ & Correlation (Pearson $\boldsymbol{r}$ ) with Pragma total score \\
\hline (a) Speech & $1.37(2.02)$ & $-0.437(p<0.001)$ \\
(b) Syntax & $0.89(1.23)$ & $-0.193(p=0.046)$ \\
(c) Semantics & $3.23(2.25)$ & $-0.302(p=0.002)$ \\
(d) Coherence & $2.65(2.53)$ & $-0.288(p=0.003)$ \\
(e) Inappropriate initiation & $5.02(3.65)$ & $-0.341(p<0.001)$ \\
(f) Stereotyped language & $2.15(1.90)$ & $-0.334(p<0.001)$ \\
(g) Use of context & $3.69(2.79)$ & $-0.425(p<0.001)$ \\
(h) Non-verbal communication & $2.04(1.97)$ & $-0.355(p<0.001)$ \\
(i) Social relationships & $1.17(1.45)$ & $-0.180(p=0.064)$ \\
(f) Interests & $5.19(3.22)$ & $-0.353(p<0.001)$ \\
\hline
\end{tabular}

Low CCC-2 raw scores denote strengths whereas low Pragma raw scores denote weaknesses. Thus, negative correlation means association between the results of these two instruments.

\subsubsection{Procedure}

The children were recruited from a number of daycare centers and primary schools in two different parts of Italy (i.e., Torino and Salerno, respectively in the north and in the south of the country). The test administrators visited the schools before the data collection began and provided teachers with a detailed description of aims and procedures of the study. A letter containing all the details about the research was sent to the children's families, together with an informed consent form, which the parents were asked to return. Only children whose families gave their written informed consent were included in the sample. The study was approved by the Bio-ethical Committee of the University of Turin (Protocol no. 23979).

\subsubsection{Inclusion criteria}

Children's linguistic skills were assessed using the language comprehension task of the NEPSY-II (Urgesi et al. 2011) and only children who performed within the normative values were included in the present study (see Table 3). Moreover, the children's caregivers were required to provide answers to seven-questions aimed at collecting information about their children's language development (e.g., at what age the child has started to use intelligible words and sentences? Did the child experience any hearing problems in the past? Did the child go through any speech therapy and for what reason?) and the children were included only if no developmental diagnosis of disorder or delay was reported. All the recruited children fulfilled the criteria and all the recruited children were included in the experimental sample. 
Table 3: Characteristics of the participants.

\begin{tabular}{lrrrrrr}
\hline Group & $\boldsymbol{N}$ & \multicolumn{2}{c}{ Gender } & Age (yrs; mths) Mean (SD) & \multicolumn{2}{c}{$\begin{array}{c}\text { NEPSY - Language } \\
\text { Comprehension task }^{\mathrm{a}}\end{array}$} \\
\cline { 3 - 7 } & & $\boldsymbol{M}$ & $\boldsymbol{F}$ & & Min-Max value & Mean (SD) \\
\hline A (4-4;11) & 22 & 8 & 14 & $4 ; 5(0 ; 35)$ & $7-13$ & $8.96(1.98)$ \\
B (5-5;11) & 22 & 13 & 9 & $5 ; 4(0 ; 32)$ & $8-14$ & $10.13(2.64)$ \\
C (6-6;11) & 22 & 11 & 11 & $6 ; 6(0 ; 29)$ & $8-14$ & $11.86(3.07)$ \\
D (7-7;11) & 22 & 10 & 12 & $7 ; 5(0 ; 38)$ & $7-14$ & $10.18(2.52)$ \\
E (8-8;11) & 22 & 12 & 10 & $8 ; 5(0 ; 32)$ & $7-12$ & $9.14(1.93)$ \\
\hline
\end{tabular}

${ }^{a}$ Scalar scores, transformed with reference to the Italian normative values for each age group.

Table 4: Correct answers of different question types by age groups.

\begin{tabular}{llrrrrr}
\hline Question type & & A & B & C & D & E \\
& & $(\mathbf{4 - 4 ; 1 1 )}$ & $\mathbf{( 5 - 5 ; 1 1 )}$ & $(6-6 ; 11)$ & $(7-7 ; 11)$ & $(\mathbf{8 - 8 ; 1 1 )}$ \\
\hline Contextual inference without & Mean & 4.91 & 6.23 & 7.7 & 8.27 & 9.09 \\
ToM demand & SD & 1.63 & 1.71 & 1.78 & 1.42 & 1.06 \\
ConNoTom $(n=10)$ & Abs. $f(\%)$ & 49.1 & 62.3 & 77.3 & 82.7 & 90.9 \\
Contextual inference with & Mean & 4.9 & 7.59 & 10.64 & 12.5 & 13.91 \\
ToM & SD & 2.39 & 2.92 & 3.43 & 3.63 & 1.92 \\
demand & Abs. $f(\%)$ & 27.3 & 42.1 & 59.1 & 69.5 & 77.3 \\
ConToM $(n=18)$ & & & & & & \\
Feelings recognition & Mean & 2.86 & 3.36 & 3.9 & 4.18 & 4.18 \\
Feeling $(n=5)$ & SD & 1.04 & 1.09 & 0.84 & 0.66 & 0.79 \\
& Abs. $f(\%)$ & 57.3 & 67.3 & 79.1 & 83.7 & 83.7 \\
False belief & Mean & 0.45 & 1.09 & 1.32 & 1.73 & 1.86 \\
FB $(n=2)$ & SD & 0.67 & 0.75 & 0.78 & 0.45 & 0.35 \\
& Abs. $f(\%)$ & 22.7 & 54.6 & 65.9 & 86.4 & 93.2 \\
Relevant language use & Mean & 0.86 & 1.63 & 1.68 & 2.0 & 2.36 \\
R.U.L. $(n=4)$ & SD & 1.04 & 1.05 & 0.84 & 1.07 & 0.79 \\
& Abs. $f(\%)$ & 21.6 & 40.9 & 42 & 50 & 59.1 \\
Pragma total score & Mean & 14 & 19.9 & 25.32 & 28.68 & 31.41 \\
$(n=39)$ & SD & 5.24 & 4.82 & 6.0 & 5.57 & 3.06 \\
& Abs. $f(\%)$ & 35.6 & 53.4 & 65.3 & 74.4 & 80.8 \\
\hline
\end{tabular}

Absolute frequency (Abs. $f$ ) represents the percentage of correct answers obtained by each age group at each question type. 


\subsubsection{Socio-economic status}

We collected information regarding family composition, parental educational level, and occupation, asking the participants' parents to fill in a questionnaire. The children's Socio-Economic Status (SES) was derived from the Four Factor Index of Social Status (Hollingshead 1975), updating the employment categories according to the current Italian social context. Each family's SES score is obtained by multiplying occupation (range 1-7) and education values (range 1-7) by 7 and 4, respectively. If both parents were employed, their indexes' mean was taken into consideration; if just one parent was employed, the final value was based on his/her index. Scores ranged from 77 (lowest SES) to 11 (highest SES). Within the present sample, $42.7 \%$ of the children were from upper-middle class families, 32.7\% from middle class, $17.3 \%$ from upper class and 3.6 \% from lower-middle class families. We verified whether our sample's SES index showed a correlation with the pragmatic performance investigated: neither Pragma total score (Pearson $r=0.47 ; p=0.634$ ) nor the General Communication Composite of the Children Communication Checklist-2 $(r=-0.100$; $p=0.31$ ) showed any correlation with the socio economic status of the participants.

\section{Results}

\subsection{Performance of the different age groups on the question tasks of the Pragma test}

First of all, we examined whether the children's performance on the Pragma test changed with age. We first analyzed the overall score obtained at the Pragma questions across the different age groups, i.e., A (4-4;11), B (5-5;11), C (6-6;11), D (7-7;11), E (8-8;11): a one-way ANOVA revealed a significant effect of age $\left(F_{(1,4)}=42.21 ; p<0.001\right.$; $\left.\eta_{p}^{2}=0.62\right)$. Post-hoc pairwise comparison showed that when comparing adjacent age groups, there was a significant difference between group A $(4-4 ; 11)$ and B $(5-5 ; 11)$ $(p=0.002 ; d=1.20)$, and between group $\mathrm{B}(5-5 ; 11)$ and group $\mathrm{C}(6-6 ; 11)(p=0.006$; $d=1.02)$. No significant differences were detected when comparing group $C(6-6 ; 11)$ and D (7-7;11) $(p=0.29 ; d=0.59)$, and group D $(7-7 ; 11)$ and $E(8-8 ; 11)(p=0.76$; $d=0.62$ ), despite the moderate effect size and despite the fact that a significant difference was detectable when comparing non adjacent groups, i.e., B (5-5;11) and D $(7-7 ; 11)(p<0.001)$ as well as Group C $(6-6 ; 11)$ with Group E $(8-8 ; 11)(p=0.001)$. See Figure 1.

The pattern of performance at different ages was then considered in relation to each question type, i.e., Contextual Inference with no ToM demand (ConNoToM), Contextual Inference with ToM demand (ConToM), Recognition of Feeling 


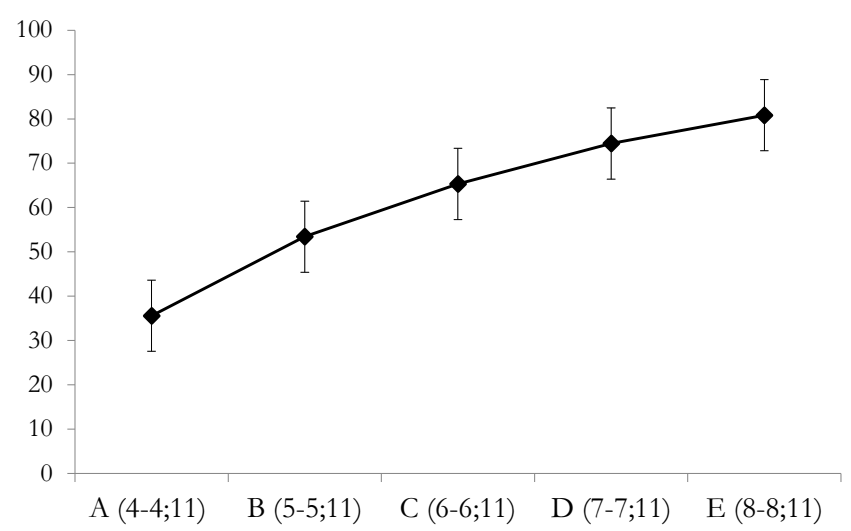

Figure 1: Mean performance scores (expressed in \%) at the Pragma test considered overall, for each age group.

(Feeling), Undersnding of False Belief (FB), and Relevant Use of Language (RUL), within each group separately. The row performance scores are displayed in Table 3 and in Figure 2 you can see a graphical representation of the trend with the scores expressed in percentage.

For the task ConNoToM, one way ANOVA showed a significant effect of age $\left(F_{(1,4)}=25.82 ; p<0.001 ; \eta_{p}^{2}=0.50\right)$. Post-hoc pairwise comparison (Bonferroni) among adjacent age groups, showed a difference at the limit of the statistical

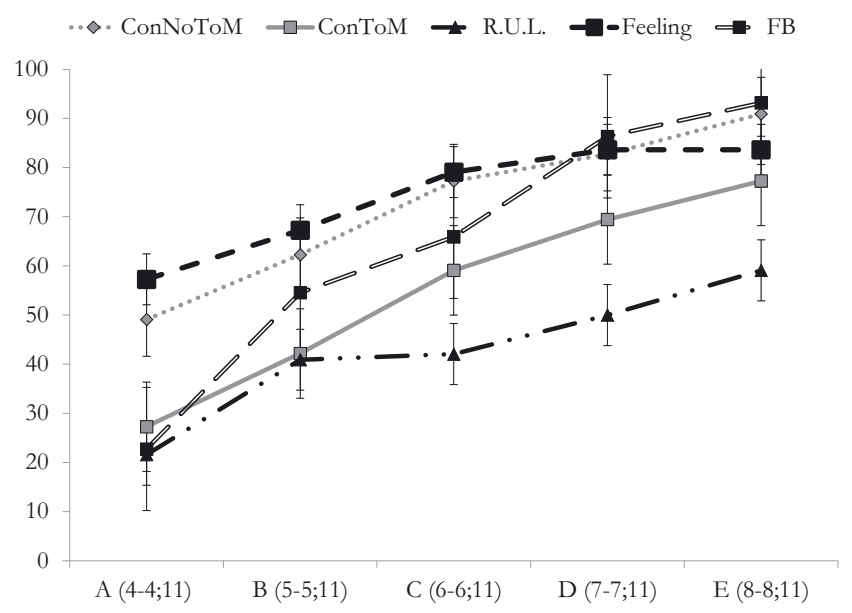

Figure 2: Mean performance scores (expressed by \%) for each age group at the different question types. 
significant between group A $(4-4 ; 11)$ and B $(5-5 ; 11)(p=0.05 ; d=0.81)$. A significant difference was detected between group B $(5-5 ; 11)$ and group $C(6-6 ; 11)$ $(p=0.017 ; d=0.88)$. No significant differences were detected when comparing group C $(6-6 ; 11)$ and $\mathrm{D}(7-7 ; 11)(p=1 ; d=0.35)$, and group $\mathrm{D}(7-7 ; 11)$ and $\mathrm{E}(8-8 ; 11)$ $(p=0.817 ; d=0.67)$. Significant differences were also detected when comparing non adjacent groups, i.e., A $(4-4 ; 11)$ and C $(6-6 ; 11)(p<0.001)$, group B $(5-5 ; 11)$ and D $(7-7 ; 11)(p<0.001)$ and group C $(6-6 ; 11)$ and $\mathrm{E}(8-8 ; 11)(p=0.042)$.

For the task ConToM, one way ANOVA showed a significant effect of age $\left(F_{(1,4)}=34.34 ; p<0.001 ; \eta_{p}^{2}=0.57\right)$. Post-hoc pairwise comparison (Bonferroni) among adjacent age groups, showed a significant difference between group $\mathrm{A}$ $(4-4 ; 11)$ and $\mathrm{B}(5-5 ; 11)(p=0.03 ; d=1.03)$. A significant difference was detected between group B $(5-5 ; 11)$ and group C $(6-6 ; 11)(p=0.008 ; d=0.98)$. No significant differences were detected when comparing group $\mathrm{C}(6-6 ; 11)$ and $\mathrm{D}(7-7 ; 11)$ $(p=0.37 ; d=0.54)$, and group D $(7-7 ; 11)$ and $\mathrm{E}(8-8 ; 11)(p=1 ; d=0.50)$. Significant differences were also detected when comparing non adjacent groups, i.e., A $(4-4 ; 11)$ and C $(6-6 ; 11)(p<0.001)$, group B $(5-5 ; 11)$ and D $(7-7 ; 11)$ $(p<0.001)$ and group C $(6-6 ; 11)$ and $\mathrm{E}(8-8 ; 11)(p=0.003)$.

As for the tasks Relevant Use of Language, one way ANOVA showed a significant effect of age $\left(F_{(1,4)}=7.31 ; p<0.001 ; \eta_{p}^{2}=0.22\right)$. Post-hoc pairwise comparison (Bonferroni) among adjacent age groups, showed no significant difference between both adjacent and non-adjacent groups $(0.05<p<1 ; 0.05<d<0.75)$.

For the task Feelings, one way ANOVA showed a significant effect of age $\left(F_{(1,4)}=9.09 ; p<0.001 ; \eta_{p}^{2}=0.26\right)$. Post-hoc pairwise comparison (Bonferroni) among adjacent age groups, showed no significant difference between groups $(0.32<p<1$; $0.01<d<0.62)$. Significant differences were detected when comparing non adjacent groups, i.e., A (4-4;11) and C (6-6;11) $(p<0.001)$, group B $(5-5 ; 11)$ and D $(7-7 ; 11)$ $(p=0.032)$, but not when comparing group $C(6-6 ; 11)$ and $\mathrm{E}(8-8 ; 11)(p=1.0)$.

For the task $F B$, one way ANOVA showed a significant effect of age $\left(F_{(1,4)}=17.73\right.$; $p<0.001 ; \eta_{p}^{2}=0.40$ ). Post-hoc pairwise comparison (Bonferroni) among adjacent age groups, showed a significant difference between group A $(4-4 ; 11)$ and $B(5-5 ; 11)$ ( $p=0.01 ; d=0.92)$, while no differences were detected when comparing the other groups $(0.32<p<1 ; 0.31<d<0.65)$. Significant differences were also detected when comparing non adjacent groups, i.e., A $(4-4 ; 11)$ and C $(6-6 ; 11)(p<0.001)$, group B $(5-5 ; 11)$ and $D(7-7 ; 11)(p=0.01)$, and group $C(6-6 ; 11)$ and $E(8-8 ; 11)(p=0.046)$.

\subsection{Performance on the different questions of the Pragma test}

We also explored in more detail the overall performance in the different question types of the Pragma test, in order to examine whether a difference in processing demand could be detected. 
The performance scores obtained by the children at each type of question, expressed in percentage, are displayed in Figure 3. Considering the sample overall, a one way ANOVA detected a significant effect of the type of task $\left(F_{(1,4)}=48.87\right.$; $\left.p<0.001 ; \eta_{p}^{2}=0.31\right)$. Children's performance was higher in Feeling and ConNoToM tasks, while the lowest performance was gained in Relevant Use of Language tasks. A simple contrast comparing the mean performance on ConNoToM tasks to the mean performance to all the others, highlighted that children performed equally well when comparing ConNoTom and Feeling tasks ( $F=0.574 ; p=0.45 ; \eta_{p}^{2}=0.005$ ), while significant differences were detectable when comparing ConNoToM with ConToM $\left(F=140.07 ; p<0.001 ; \eta_{p}^{2}=0.56\right)$, ConNoToM with R.U.L. $(F=177.65$; $\left.p<0.001 ; \eta_{p}^{2}=0.62\right)$ and ConNoToM with $F B\left(F=6.87 ; p=0.01 ; \eta_{p}^{2}=0.06\right)$.

\subsection{Performance on the explanation tasks of the Pragma test}

Finally, we examined the children's performance on the explanation tasks. The number of correct explanations is displayed in Figure 4, together with the number of correct answers to the question the explanation refers to (the explanation was asked and scored as correct only when the question it referred to was first answered correctly).

The analysis of the relative frequency (number of correct explanations $/ f \times 100 \%$ ) showed that children belonging to group A $(4-4 ; 11)$ were able to successfully explain $48.2 \%$ of their correct answers, children belonging to group B $(5-5 ; 11)$ the $48.21 \%$, children of group C (6-6;11) $65.73 \%$, children of group D $(7-7 ; 11) 72.5 \%$ and children

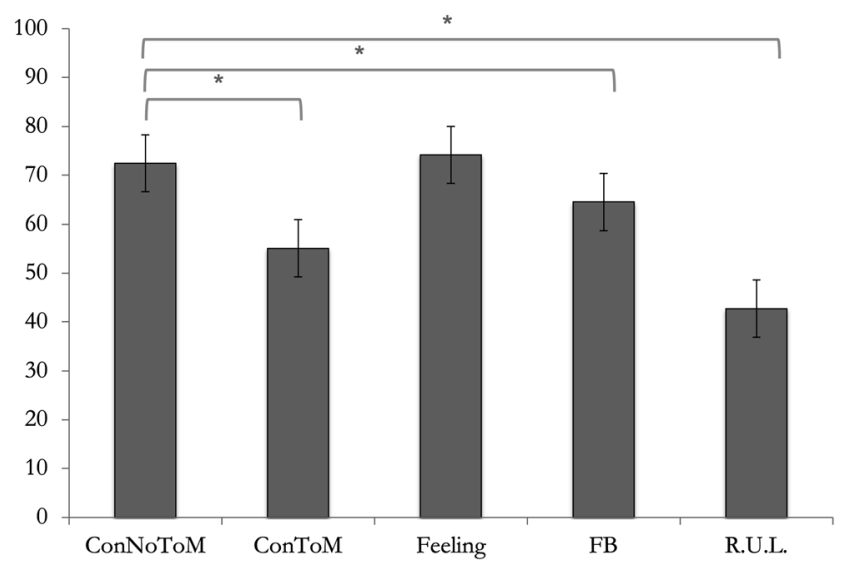

Figure 3: Overall performance (expressed in \%) at the different question types of the Pragma test. 


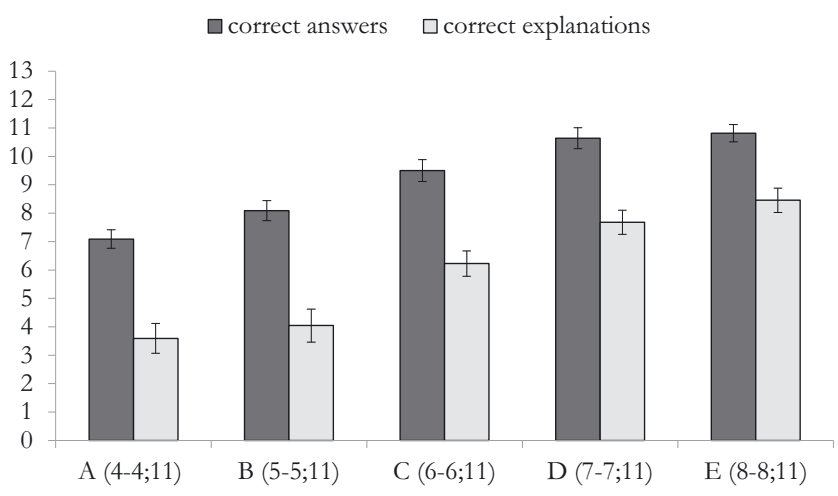

Figure 4: Mean score of correct explanations and relative answers $(N=13)$ in each age group.

of group E $(8-8 ; 11) 77.9 \%$ of their correct answers. A one-way unrelated ANOVA revealed a significant effect of age in children's ability to provide an explanation for their correct answers $\left(F_{(1,4)}=19.73 ; p<0.001 ; \eta_{p}^{2}=0.43\right)$. According to a post-hoc comparison (Bonferroni), this seemed to be mostly due to the difference in performance between children belonging to group $B(5-5 ; 11)$ and children belonging to group C (6-6;11) ( $p=0.016)$.

\subsection{Sex differences in the performance on the Pragma Tasks}

We have also examined, with independent samples $t$-test analysis, whether any difference was detectable in the performance scores obtained by males and females at the Pragma tasks (see Figure 5). The results on the overall sample indicate no differences both regarding the scores obtained at the questions $(t=0.098 ; p=0.922 ; d=0.02)$ and at the explanation $(t=1.212 ; p=0.228 ; d=0.15)$ tasks of the Pragma test.

\section{Discussion}

This study explored data on pragmatic performance of typically developing Italian children using the Italian adaptation of the Pragma test (Loukusa 2019; Loukusa et al. 2017) which was originally developed in Finland to assess children's socialpragmatic comprehension. Adapting the Finnish Pragma test into the Italian context has a number of advantages, including the enabling of the comparison of the results of different studies, allowing cross cultural comparison, and reducing the crowding effect when it comes to research groups working on similar topics. 


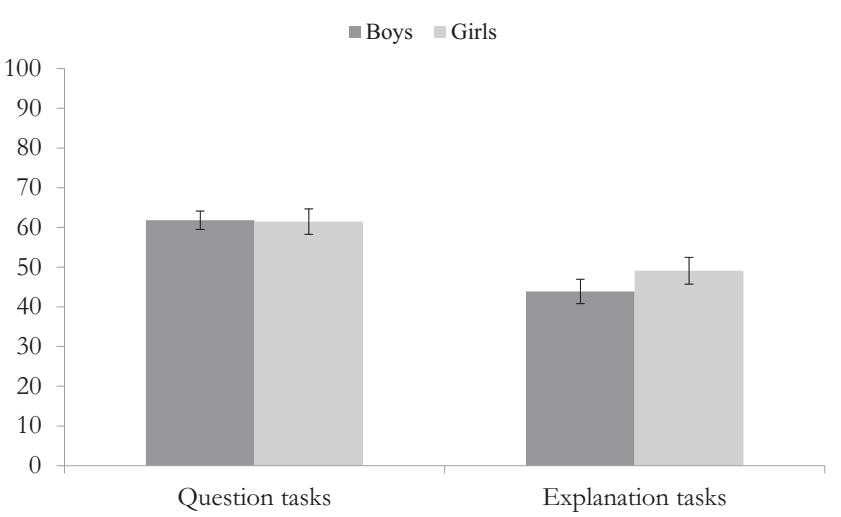

Figure 5: Mean performance scores (expressed in \%) at the question and explanation tasks of the Pragma test considered overall, obtained by boys and girls.

However, the adaptation of a tool for pragmatic assessment is quite challenging due to linguistic and cultural differences (see Bornman et al. 2010).

In this study we focused on typically developing Italian children ranging from four to eight years of age. The Italian version of the Pragma test has proven to be sensitive in detecting a developmental pattern, across pre-school and school age children, in the pragmatic parameters that were investigated. Moreover, the test has shown to have content validity, interrater reliability and internal consistency. The study has also shown a correlation between the total score of the Pragma test and the General Communication Composite of the CCC-2 (Bishop et al. 2003). Moreover, significant correlation was detected when considering the scores at each of the scales of the CCC-2, with the one exception, (i) social relation, but that is close to the statistical significance. The CCC-2 is a tool widely used particularly in the clinical settings for the screening of communication problems in children and adolescents and used in the present study as a measure of concurrent validity. The results show that higher score at the Pragma test correspond to a fewer reported communicative difficulties in the CCC-2, thus suggesting a good sensitivity of the Pragma test in detecting pragmatic difficulties in line with previous studies where the Pragma test has been used with clinical populations (see Loukusa et al. 2018). The two assessment methods can be seen to be complimentary, due to a focus on similar but not entirely overlapping aspects of communication, as well as to their totally different structure (direct administration protocol in one case and screening questionnaire filled in by a caregiver in the other one). Overall, our study shows that despite linguistic differences it is possible to successfully adapt assessment tools developed in different cultural context. 
Participants came from two different parts of Italy (i.e., Torino and Salerno, located in the north and south of Italy, respectively) and were stratified according to the index of Socio Economic Status (SES; Hollingshead 1975). Our data showed that no correlation was found between the families' socio economic status and the children's communicative performance, which is in line with previous studies (Bosco et al. 2013; Bosco and Gabbatore 2016a, 2016b), showing that SES had only a moderate overall effect on children's pragmatic performance during the schooling period.

Social-pragmatic abilities are of great importance in an individual's life due to the role they play in children's interaction with other people, as well as their relationship to social norms and social engagement in the community (see Tomasello 2019 for an in-depth discussion). The assessment of such skills is, then, of a great importance in pre-school and school age, as social-pragmatic skills are commonly required during the various communicative interaction children are engaged in everyday, at home and at school, including the effect that communication has on building relationships with peers (Gertner et al. 1994) as well as with teachers (Edwards and Mercer 1986; see also Grigoroglou and Papafragou 2017). Experiencing pragmatic difficulties can result in poor peers interactions (Conti-Ramsden and Botting 2004; Mackie and Law 2010; Whitehouse et al. 2009), as well as behavioral, social, and emotional problems (e.g., St Clair et al. 2011). Gaining clarity about typical developmental pattern as well as strengths and weaknesses at different ages, helps professionals monitor children's well-being and intervene whenever needed. Moreover, data on typical development is critical when assessing pragmatic abilities of children with developmental disorders characterized by pragmatic impairments, as in the case of ASD (Angeleri et al. 2016; Loukusa and Moilanen 2009; Loukusa et al. 2018), ADHD (Loukusa 2017b; Väisänen et al. 2014), or children with hearing impairment (O'Reilly et al. 2014).

This study found that, in line with our hypothesis, a difference in performance could be detected in the different age groups: the data suggest that the youngest children, 4 years of age, found it difficult to answer pragmatically complex questions, and that performance improved with age, without reaching a ceiling effect. The improvement in the performance scores was particularly significant from 4-6 years of age and slows down around the age of 7 . No significant difference was found when comparing 6 and 7 year olds, as well as 7 and 8 year olds, but the differences were strongly significant when comparing the performance of children of 6 and 8 years of age. This shows a slower but still detectable improvement in the abilities. Such a pattern of results in pragmatic performance as a function of age is in line with previous studies (e.g., Angeleri and Airenti 2014; Bosco et al. 2004; Bosco and Gabbatore 2017a, 2017b; Loukusa et al. 2007, 2008). With age, children learn how to correctly interpret utterances and communicative acts by directing their attention 
towards relevant sources of contextual information (Loukusa et al. 2008; Ryder and Leinonen 2003) and to express complex issues explicitly (Mäkinen et al. 2014).

Moreover, pragmatic ability is supported by a number of social and cognitive skills, which also develop rapidly in this age range. Even if ToM skills still develop across the school age (e.g., Wellman and Liu 2004), and continue to improve across adolescence (Bosco et al. 2014; Brizio et al. 2015), both ToM and cognitive abilities such as self-monitoring and cognitive flexibility appear to improve rapidly between the ages of 4 and 6 (Wellman et al. 2001; Wimmer and Perner 1983), which might partly be due to socialization with adults and peers in pre-school and school contexts. This may then partially explain why greatest acceleration in development of pragmatic performance has been observed between the ages of 4 and 6 . The performance of children in the older age groups didn't show statistically significant differences.

When looking at the pattern of performance of the children in the different age groups for each question type, we note that children obtain higher scores when processing contextual inferences without ToM consideration (ConNoTom) than when processing contextual inferences with ToM demand (ConTom). As for the Relevant Use of Language and Feeling tasks, despite the detection of a general effect of age, no significant differences were found when comparing adjacent age groups. For both types of tasks the variability might be lower across the age groups as these are, respectively, the easiest and the most difficult abilities in processing terms for the child. Some studies show that the recognition of simple feelings develops early, as conveyed by facial expressions and eye gaze (Hoehl and Striano 2010; see also Yuill and Little 2018). This is reflected in the Pragma test performance in this study by the youngest children. However, the Relevant Use of Language tasks were quite challenging for all the children in this study, which may partially explain a small variability across the age groups. Some studies suggest that children develop social norms quite easily (see Diesendruck and Markson 2011; Rakoczy and Schmidt 2013), they mostly refer to conventionality in game interaction with peers and adults. This study suggests that understanding more general social norms in a variety of contexts was required to perform well. Our data suggest that the development of the use of language in different contexts is evident in our data but this is developing slower than the other abilities investigated with the Pragma test, when comparing adjacent age groups rather than when comparing age groups two years apart from each other.

A significant difference was detected in relation to the questions examining False Belief when comparing the performance of 4 and 5 years olds. This is in line with studies indicating that false belief tasks are mastered around the age of 4 (Wellman et al. 2001; Wimmer and Perner 1983). As for the older children, a 
significant increase in performance was detected only when comparing non adjacent groups.

The present study also presents interesting findings regarding the children's performance in the different types of questions. Considering the sample overall, the children obtained higher scores in Feeling and ConNoToM questions, reflecting the ability to recognize simple emotions and making contextual inferences that do not require ToM ability. At the opposite end, children obtained the lowest scores in Relevant Use of Language and ConToM questions reflecting the ability to draw variety of contextual information in the particular contexts and children's ability to make contextual inferences when requiring to consider the mental states of others. As expected, the analysis revealed that the scores obtained at the ConNoToM questions were higher than those children obtained at False Belief and ConToM tasks. This result is in line with that of Loukusa et al. (2017), a study which examined the same abilities in Finnish typically developing children of the same age. This suggests that understanding and taking into consideration mental states such as intentions and (false)beliefs of other people, represent an additional level of complexity when compared to the ability to make inferences from different contextual sources only, without the necessity to consider the mental states of others. This result also supports the idea that pragmatic ability and ToM are two distinct capacities interacting but not overlapping (see Bosco et al. 2018).

A further aim of the study was to focus not only on the children's ability to answer pragmatically complex questions, but also their awareness of their own reasoning leading to their correct answers. This aspect has not been much investigated in previous literature, however a few studies have suggested that such metacognitive ability increases significantly between the ages of four and eight (Letts and Leinonen 2001; Loukusa et al. 2017, 2008). In this study, between the ages of five and six a difference was identified in Italian children's ability to give appropriate explanations for their correct answers (five-year-olds explained $48 \%$ and six-year-olds $66 \%$ of their answers correctly). Although six-year-old children managed to explain many of their correct answers, this ability was even more evident in children at the age of eight, reaching $78 \%$ correct explanations. It therefore appears that it takes many years for children to became competent to explicitly express the reasoning which underpin their correct answers (see also Donaldson 2006; Letts and Leinonen 2001; Loukusa et al. 2017, 2008).

Moreover, it is interesting to note that despite the fact that the assessment tool was specifically developed for children, no ceiling effect was detected, in line with the developmental pattern detected so far in Finnish children. Our data indicates a need to investigate pragmatic comprehension from middle childhood to adolescence, as the development of the pragmatic abilities investigated within the present study seems to slow down, but not come to a ceiling effect at age 7-8. 
Finally, the exploration of sex differences in the pragmatic performance as detected by the tasks of the Pragma test, revealed that boys and girls had similar performance. This result is similar to that of Loukusa et al. (2007) where no gender differences were found in pragmatic scores of children aged 3-9 years. Moreover, this is in line with a very recent review and meta-analysis on the neurobiology of sex differences (Sato 2020), whose results suggest a higher degree of similarities rather than differences in the human brain of males and female during language processing.

The complex relationship between pragmatics, ToM and other cognitive functions is difficult to disentangle both in typical (see Bosco e Gabbatore 2017a, 2017b) and atypical populations (Cardillo et al. 2020; Parola et al. 2020; see also Matthews et al. 2018 for a review) and might imply a range of variability only partially reflected in the performance exhibited in lab settings. Due to their interconnectedness, ToM and pragmatic abilities may vary more during spontaneous interactions, and based on the communicative situation itself and the involved interlocutors (see also Tantucci 2020). However, the tasks composing the Pragma test are considered to reflect real-life social-pragmatic demands in a structured test situation. This enables the identification of potential difficulties in pragmatic, related ToM and cognitive abilities.

Our study further shows that pragmatic ability examined by the Pragma test displays similar evidence of age effects in Italian and Finnish contexts and the Pragma test is a useful tool in both cultural contexts. The Test was successfully adapted to the different cultural contexts and provides the opportunity to make cross-linguistic and cross-cultural comparisons (Gabbatore et al. 2019). Culture and communication are interconnected, as culture provides the contextual backdrop for language and communication (Schröder 2010). Studies have highlighted that social and cultural norms influence communication as is the case in making requests (Cohen and Olshtain 1993), giving advice (Matsumura 2001), or using referential communication and politeness (Fukushima and Sifianou 2017). Cultural differences may be detected also within the European context (e.g., Huttunen et al. 2013), and between cultural contexts like Finnish and Italian that, despite relative geographical and social proximity, are characterized by different communicative norms (see Gabbatore et al. 2019; Mäkinen et al. 2020).

The present study has implications for clinical contexts, for identification and management of a number of clinical populations. Availability of data regarding pragmatic abilities in typical development helps to identify strengths and weaknesses in the pragmatic performance of different clinical populations, facilitating the creation of appropriate clinical interventions (see for example Bosco et al. 2016; Gabbatore et al. [under review]; Gabbatore et al. 2015). Further studies with larger 
samples as well as a longitudinal assessment are desirable in order to consolidate the present results and allow for further and more fine-grained analyses.

Acknowledgments: This research was financially supported by the Academy of Finland (Decision number: 333672) and by Compagnia di San Paolo: Bando Ex-post University of Turin (Grant Number: D11G19000220007). The authors would like to express their gratitude to the children participating in the study, and to their families and teachers.

\section{References}

Adams, Catherine, Elaine Lockton, Jaqueline Gaile \& Jenny. Freed. 2011. TOPICCAL applications: Assessment of children's conversation skills. Speech and Language Therapy in Practice. Spring. 7-9.

Angeleri, Romina, Francesca M. Bosco, Marina Zettin, Katiuscia Sacco, Livia Colle, \& Bruno G. Bara. 2008. Communicative impairment in traumatic brain injury: A complete pragmatic assessment. Brain and Language 107(3). 229-245.

Angeleri, Romina \& Gabriella Airenti. 2014. The development of joke and irony understanding: A study with 3- to 6-year-old children. Canadian Journal of Experimental Psychology 68(2). 133-146.

Angeleri, Romina, Francesca M. Bosco, Ilaria Gabbatore, Bruno G. Bara \& Katiuscia Sacco. 2012. Assessment battery for communication ( $\mathrm{ABaC}$ ): Normative data. Journal of Behavior Research Methods 44(3). 845-861.

Angeleri, Romina, Ilaria Gabbatore, Francesca M. Bosco, Katiuscia Sacco \& Livia Colle. 2016. Pragmatic abilities in children and adolescents with autism spectrum disorder: A study with the ABaCo battery. Minerva Psichiatrica 57(3). 93-103.

Apperly, Ian. 2010. Mindreaders: the cognitive basis of "theory of mind". New York: Psychology Press.

Bauer, Daniel J., Beverly A. Goldfield, \& Steven J. Reznick. 2002. Alternative approaches to analyzing individual differences in the rate of early vocabulary development. Applied PsychoLinguistics 23. 313-335.

Bishop, Dorothy V. M. 2003. The children's communication checklist - 2. London, UK: The Psychological Corporation.

Bornman, Juan, Rose A. Sevcik, Mary Ann Romski \& Pae Hye Kyeong. 2010. Successfully translating language and culture when adapting assessment measures. Journal of Policy and Practice in Intellectual Disabilities 7(2). 111-118.

Bosco, Francesca M., Romina Angeleri, Livia Colle, Katiuscia Sacco \& Bruno G. Bara. 2013. Communicative abilities in children: An assessment through different phenomena and expressive means. Journal of Child Language 40(4). 741-778.

Bosco, Francesca M., Romina Angeleri, Marco Zuffranieri, Bruno G. Bara \& Katiuscia Sacco. 2012. Assessment battery for communication: Development of two equivalent forms. Journal of Communication Disorders 45(4). 290-303.

Bosco, Francesca M., Monica Bucciarelli \& Bruno G. Bara. 2004. The fundamental context categories in understanding communicative intention. Journal of Pragmatics 36. 467-488. 
Bosco, Francesca M. \& Ilaria Gabbatore. 2017a. Sincere, deceitful, and ironic communicative acts and the role of the theory of mind in childhood. Frontiers in Psychology 8. 21.

Bosco, Francesca M. \& Ilaria Gabbatore. 2017b. Theory of mind in recognizing and recovering communicative failures. Applied PsychoLinguistics 38(1). 57-88.

Bosco, Francesca M., Ilaria Gabbatore, Luigi Gastaldo \& Katiuscia Sacco. 2016. Communicative pragmatic treatment in schizophrenia: A pilot study. Frontiers in Psychology 7.166.

Bosco, Francesca M., Ilaria Gabbatore \& Tirassa Maurizio. 2014. A broad assessment of theory of mind in adolescence: The complexity of mindreading. Consciousness and Cognition 24. 84-97.

Bosco, Francesca M., Ilaria Gabbatore, Maurizio Tirassa \& Silvia Testa. 2016. Psychometric properties of the theory of mind assessment scale in a sample of adolescents and adults. Frontiers in Psychology 7. 1-12.

Bosco, Francesca M., Maurizio Tirassa \& Ilaria Gabbatore. 2018. Why pragmatics and theory of mind do not (Completely) overlap. Frontiers in Psychology 9. 1-7.

Brizio, Adelina, Ilaria Gabbatore, Maurizio Tirassa \& Francesca M. Bosco. 2015. “No more a child, not yet an adult": Studying social cognition in adolescence. Frontiers in Psychology 6. 1011.

Cardillo, Ramona, Irene C. Mammarella, Ellen Demurie, David Giofrè \& Herbert Roeyers. 2020. Pragmatic language in children and adolescents with autism spectrum disorder: Do theory of mind and executive functions have a mediating role? Autism Research.1-14. https://doi.org/ 10.1002/aur.2423.

Cheung, Him, Tik-Sze Siu \& Lan Chen. 2015. The roles of liar intention, lie content, and theory of mind in children's evaluation of lies. Journal of Experimental Child Psychology 132. 1-13.

Cohen, Andrew D. \& Elite Olshtain. 1993. The production of speech acts by EFL learners. Tesol Quarterly 27(1). 33.

Colle, Livia, Romina Angeleri, Marianna Vallana, Katiuscia Sacco, Bruno G. Bara \& Francesca M. Bosco. 2013. Understanding the communicative impairments in schizophrenia: A preliminary study. Journal of Communication Disorders 46. 294-308.

Colletta, Jean-Marc, Michele Guidetti, Olga Capirci, Carla Cristilli, Ozlem E. Demir, Ramona N. Kunene-Nicolas \& Susan Levine. 2014. Effects of age and language on co-speech gesture production: An investigation of French, American, and Italian children's narratives. Journal of Child Language 96(1). 122-145.

Conti-Ramsden, Gina \& Nicola Botting. 2004. Social difficulties and victimization in children with SLI at 11 years of age. Journal of Speech, Language, and Hearing Research 47(1). 145-161.

Cummings, Luise. 2009. Clinical pragmatics. Cambridge, UK: Cambridge University Press.

Diesendruck, Gil \& Lori Markson. 2011. Children's assumption of the conventionality of culture. Child Development Perspectives 5(3). 189-195.

Di Sano, Sergio, Aristide Saggino, Maria Silvia Barbieri, Marco Tommasi \& Luca Surian. 2013. Children Communication Checklist. Second Edition versione italiana. Firenze: Giunti OS Organizzazioni Speciali.

Donaldson, Morgan L. 2006. Children's explanations: A psycholinguistic study. Cambridge, UK: Cambridge University Press.

Edwards, Derek. \& Neil Mercer. 1986. Context and continuity: Classroom discourse and the development of shared knowledge. In Durkin, K. (ed.), Language Development in School Years. 172-203. London: Croom Helm.

Fukushima, Saeko \& Maria Sifianou. 2017. Conceptualizing politeness in Japanese and Greek. Intercultural Pragmatics 14(4). 525-555. 
Gabbatore, Ilaria, Romina Angeleri, Francesca M. Bosco, Federico M. Cossa, Bruno G. Bara \& Katiuscia Sacco. 2014. Assessment of communicative abilities in aphasic patients. Minerva Psichiatrica 55(2). 45-55.

Gabbatore, Ilaria, Francesca M. Bosco, Leena Mäkinen, Ebeling Hanna, Tuula Hurtig \& Soile Loukusa. 2019. Investigating pragmatic abilities in young Finnish adults using the Assessment Battery for Communication. Intercultural Pragmatics 16(1). 27-56.

Gabbatore, Ilaria, Claudio Longobardi \& Francesca M. Bosco. Under review. Improvement of communicative-pragmatic ability in adolescents with Autism Spectrum Disorder: The adapted version of the Cognitive Pragmatic Treatment. Language Learning and Development.

Gabbatore, Ilaria, Katiuscia Sacco, Romina Angeleri, Marina Zettin, Bruno G. Bara \& Francesca M. Bosco. 2015. Cognitive pragmatic treatment: A rehabilitative program for traumatic brain injury individuals. The Journal of Head Trauma Rehabilitation 30(5). E14-E28.

Gertner, Bethany L., Mabel L. Rice \& Pamela A. Hadley. 1994. Influence of communicative competence on peer preferences in a preschool classroom. Journal of Speech, Language, and Hearing Research 37. 913-923.

Grigoroglou, Myrto \& Anna Papafragou. 2017. Acquisition of pragmatics. In M. Aronoff (ed.), Oxford research encyclopedia of linguistics, 1-39. Oxford: Oxford University Press.

Hoehl, Stefanie \& Tricia Striano. 2010. The development of emotional face and eye gaze processing. Developmental Science 13(6). 813-825.

Hoff-Ginsberg, Erika. 1998. The relation of birth order and socioeconomic status to children's language experience and language development. Applied Psycholinguistics 19(4). 603-629.

Hollingshead, August B. 1975. Four Factor Index of Social Status. New Haven, Conn.: Yale University.

Huttunen, K. H., K. J. Pine, A. J. Thurnham \& C. Khan. 2013. The changing role of gesture in linguistic development: A developmental trajectory and a cross-cultural comparison between British and Finnish children. Journal of Psycholinguistic Research 42(1). 81-101.

Küntay, Aylin, Keiko Nakamura \& Beyza Ateş Sen. 2014. Crosslinguistic and crosscultural approaches to pragmatic development. In Danielle Matthews (ed.), Pragmatic development in first language acquisition, 317-342. Amsterdam: John Benjamins.

Lecce, Serena, Luca Ronchi, Paola Del Sette, Luca Bischetti \& Valentina Bambini. 2019. Interpreting physical and mental metaphors: Is Theory of Mind associated with pragmatics in middle childhood? Journal of Child Language 46(2). 393-407.

Leinonen, Eeva, Christophe Letts \& Brent R. Smith. 2000. Children's pragmatic communication difficulties. London: Whurr.

Letts, Christophe \& Eeva Leinonen. 2001. Comprehension of inferential meaning in languageimpaired and language normal children. International Journal of Language \& Communication Disorders 36(3). 307-328.

Levinson, Stephen C. 1983. Pragmatics. Cambridge, UK: Cambridge University Press.

Loukusa, Soile. 2019. Pragma-testi. Jyväskylä. Finland: Niilo Mäki -Instituutti.

Loukusa, Soile. 2017a. Attention deficit hyperactivity disorder. In Luise Cummings (ed.), Research in clinical pragmatics, Series: Perspectives in pragmatics, philosophy \& psychology, Vol. 11. Cham, Switzerland: Springer-Verlag.

Loukusa, Soile. 2017b. Attention deficit hyperactivity disorder. In Perspectives in pragmatics, philosophy and psychology, Vol. 11, 85-107. Springer International Publishing.

Loukusa, Soile \& Eeva Leinonen. 2008. Development of comprehension of ironic utterances in 3- to 9-year-old Finnish-speaking children. Psychology of Language and Communication 12(1). 55-69. 
Loukusa, Soile, Eeva Leinonen \& Nuala Ryder. 2007. Development of pragmatic language comprehension in Finnish-speaking children. First Language 27(3). 279-296. https://doi. org $/ 10.1177 / 0142723707076568$.

Loukusa, Soile, Leena Mäkinen, Ilaria Gabbatore, Paivi Laukkanen-Nevala \& Eeva Leinonen. 2017. Understanding contextual and social meaning in typically developing finnishspeaking fourto eight-year-old children. Psychology of Language and Communication 21(1). 408-428.

Loukusa, Soile, Leena Mäkinen, Ilaria Gabbatore, Krista Wallenius, Hanna Ebeling \& Eeva Leinonen. 2019. Social and pragmatic inference in typical development, autism spectrum disorder and developmental language disorder as assessed by the Pragma Test. 31st World Congress of the IALP. The Abstract Book of Oral Presentations, 18-22 August 2019, Taipei, Taiwan.

Loukusa, Soile, Leena Mäkinen, Sanna Kuusikko-Gauffin, Hanna Ebeling \& Eeva Leinonen. 2018. Assessing social-pragmatic inferencing skills in children with autism spectrum disorder. Journal of Communication Disorders 73. 91-105.

Loukusa, Soile \& Irma Moilanen. 2009. Pragmatic inference abilities in individuals with Asperger syndrome or high-functioning autism. A review. Research in Autism Spectrum Disorders 3(4). 890-904.

Loukusa, Soile \& Nuala Ryder. 2007. Development of pragmatic language comprehension in Finnish-speaking children. First Language 27(3). 279-296.

Loukusa, Soile, Nuala Ryder \& Eeva Leinonen. 2008. Answering questions and explaining answers: A study of finnish-speaking children. Journal of Psycholinguistic Research 37(3). 219-241.

Mackie, Leila \& James Law. 2010. Pragmatic language and the child with emotional/behavioural difficulties (EBD): A pilot study exploring the interaction between behaviour and communication disability. International Journal of Language \& Communication Disorders 45. 397-410.

Mäkinen, Leena, Ilaria Gabbatore, Soile Loukusa, Sari Kunnari \& Phyllis Schneider. 2020. A comparison of picture-based narratives by Finnish, Italian and English-speaking children. Early Education \& Development 31(3). 395-410.

Mäkinen, Leena, Soile Loukusa, Lea Nieminen, Eeva Leinonen \& Sari Kunnari. 2014. The development of narrative productivity, syntactic complexity, referential cohesion and event content in four- to eight-year-old Finnish children. First Language 34(1). 24-42.

Maurizio, Tirassa \& Francesca M. Bosco. 2008. On the nature and role of intersubjectivity in human communication. Emerging Communication: Studies in New Technologies and Practices in Communication 10. 81-95.

Matsumura, Shoichi. 2001. Learning the rules for offering advice: A quantitative approach to second language socialization. Language Learning 51(4). 635-679.

Matthews, Danielle. 2014. Pragmatic development in first language acquisition. Amsterdam/ Philadelphia: John Benjamins Publishing Company.

Matthews, Danielle, Hannah Biney \& Kirsten Abbot-Smith. 2018. Individual differences in children's pragmatic ability: a review of associations with formal language, social cognition, and executive functions. Language Learning and Development 14(3). 186-223.

Murray, Ann D., Johnson Jeanne \& Jo Peters. 1990. Fine-tuning of utterance length to preverbalinfants: Effects on later language development. Journal of Child Language 17. 511-525. 
Nilsen, Elisabeth S., Melanie Glenwright \& Vanessa Huyder. 2011. Children and adults understand that verbal irony interpretation depends on listener knowledge. Journal of Cognition and Development 12(3). 374-409.

Norbury, Courtenay F., Marysia Nash, Gillian Baird \& Dorothy Bishop. 2004. Using a parental checklist to identify diagnostic groups in children with communication impairment: A validation of the Children's Communication Checklist-2. International Journal of Language \& Communication Disorders 39(3). 345-364.

O'Neill, Daniela K. 2012. Components of pragmatic ability and children's pragmatic language development. In H.-J. Schmid (ed.), Cognitive pragmatics, 261-287. Berlin: De Gruyter.

O’Neill, Daniela K. 2014. Assessing pragmatic language functioning in young children: Its importance and challenges. In D. Matthews (ed.), Pragmatic development in first language acquisition, 363-386. Amsterdam: John Benjamins.

O’Reilly, Karin, Candida C. Peterson \& Henrly M. Wellman. 2014. Sarcasm and advanced theory of mind understanding in children and adults with prelingual deafness. Developmental Psychology 50(7). 1862-1877.

Parola, Alberto, Ilaria Gabbatore, Francesca M. Bosco, Bruno G. Bara, Federico M. Cossa, Patrizia Gindri \& Katiuscia Sacco. 2016. Assessment of pragmatic impairment in right hemisphere damage. Journal of Neurolinguistics 39. 10-25.

Parola, Alberto, Rogerio Salvini, Ilaria Gabbatore, Livia Colle, Laura Berardinelli \& Francesca M. Bosco. 2020. Pragmatics, theory of mind and executive functions in schizophrenia: Disentangling the puzzle using machine learning. PLoS One 15(3). e0229603.

Perkins, Michael R. 2007. Pragmatic impairments. Cambridge: Cambridge University Press.

Phelps-Terasaki, Diana \& Tricha Phelps-Gunn. 2007. TOPL-2: Test of Pragmatic Language, 2nd edn. Austin, TX: Pro Ed.

Phillips, Jonathan, Wesley Buckwalter, Fiery Cushman, Ori Friedman, Alia Martin, John Turri, Laurie Santos \& Joshua Knobe. 2020. Knowledge before belief. Behavioral and Brain Sciences. 1-37. https://doi.org/10.1017/s0140525x20000618.

Premack, David \& Guy Woodruff. 1978. Does the chimpanzee have a theory of mind? The ehavioral and Brain Sciences 34(1). 1401-1407.

Rakoczy, Hannes \& Marco F. H. Schmidt. 2013. The Early Ontogeny of Social Norms. Child Development Perspectives 7(1). 17-21.

Roulstone, Sue, Sue Loader \& Kate Northstone. 2002. Descriptive data from the Avon longitudinal study of parents and children. Early Child Development and Care 22. 259-268.

Row, Meredith L. 2008. Child-directed speech: Relation to socioeconomic status, knowledge of child development and child vocabulary skill. Journal of Child Language 35. 185-205.

Ryder, Nuala \& Eeva Leinonen. 2003. Use of context in question answering by 3-, 4-and 5-year-old children. Journal of Psycholinguistic Research 32(4). 397-415. https://doi.org/10.1023/a: 1024847529077.

Ryder, Nuala, Eeva Leinonen \& Joerg Schulz. 2008. Cognitive approach to assessing pragmatic language comprehension in children with specific language impairment. International Journal of Language \& Communication Disorders 43(4). 427-447.

Sato, Marc. 2020. The neurobiology of sex differences during language processing in healthy adults: A systematic review and a meta-analysis. Neuropsychologia 140. 107404.

Saxe, Rebecca. 2013. The new puzzle of theory of mind development. In M. R. Banaji \& S. A. Gelman (eds.), The development of navigating the social cognition. What infants, children, and other species can teach us, 107-122. New York: Oxford University Press. 
Schröder, Ulrike. 2010. Speech styles and functions of speech from a cross-cultural perspective. Journal of Pragmatics 42(2). 466-476.

Searle, John R. 1975. Indirect speech acts. In P. Cole \& J. L. Morgan (eds.), Syntax and Semantics. Volume 3: Speech Acts, 59-82. San Diego, CA: Academic Press.

Shrout, Patrick E. 1998. Measurement reliability and agreement in psychiatry. Statistical Methods in Medical Research 7. 301-317.

Sodian, Beate \& Uta Frith. 1992. Deception and sabotage in autistic, retarded and normal children. Journal of Child Psychology and Psychiatry 33(3). 591-605.

Sperber, Dan \& Deirdre Wilson. 1995. Relevance: Communication and cognition, 2nd edn. Oxford: Basil Blackwell.

Sperber, Deirdre \& Dan Wilson. 2012. Introduction: pragmatics. In D. Wilson \& S. Sperber (eds.), Meaning and relevance, 1-27. Cambridge: Cambridge University Press.

St Clair, Michelle C., Andrew Pickles, Durkin Kevin \& Gina Conti-Ramsden. 2011. A longitudinal study of behavioral, emotional and social difficulties in individuals with a history of specific language impairment (SLI). Journal of Communication Disorders 44(2). 186-199.

Sullivan, Kate, Ellen Winner \& Natalie Hopfield. 1995. How children tell a lie from a joke: The role of second-order mental state attributions. British Journal of Developmental Psychology 13(2). 191-204.

Tantucci, Vittorio. 2020. From co-actionality to extended intersubjectivity: Drawing on language change and ontogenetic development. Applied Linguistics 41(2). 185-214.

Tantucci, Vittorio \& Aiqing Wang. 2020. From co-actions to intersubjectivity throughout Chinese ontogeny: A usage-based analysis of knowledge ascription and expected agreement. Journal of Pragmatics 167. 98-115.

Tomasello, Michael. 2019. Becoming human: A theory of ontogeny. Cambridge, MA: Harvard University Press.

Urgesi, Cosimo, Fabio Campanella \& Franco Fabbro. 2011. NEPSY-II. Second Contributo alla taratura italiana (NEPSY-II. Second Italian Standardization). Firenze, Italy: Giunti O.S. Organizzazioni Speciali.

Väisänen, Raija, Soile Loukusa, Irma Moilanen \& Anneli Yliherva. 2014. Language and pragmatic profile in children with ADHD measured by Children's communication checklist $2^{\text {nd }}$ edition. Logopedics Phoniatrics Vocology 39(4). 179-187.

Wellman, Hanry M., David Cross \& Julanne Watson. 2001. Meta-analysis of theory-of-mind development: The truth about false belief. Child Development 72(3). 655-684.

Wellman, Hanry M. \& David Liu. 2004. Scaling of Theory-of-Mind Tasks. Child Development 75(2). 523-541.

Whitehouse, Andrew J. O., Helen J. Watt, E.A. Line \& Dorothy H. Bishop. 2009. Adult psychosocial outcomes of children with specific language impairment, pragmatic language impairment and autism. International Journal of Language \& Communication Disorders 44(4). 511-528.

Wiig, Elisabeth \& Wayne Secord. 2014. CELF 5: Metalinguistics. Bloomington, MN: Pearson.

Wimmer, Heinz \& Josef Perner. 1983. Beliefs about beliefs: Representation and constraining function of wrong beliefs in young children's understanding of deception. Cognition 13(1). 103-128. 
Winner, Ellen \& Sue Leekam. 1991. Distinguishing irony from deception: Understanding the speaker's second-order intention. British Journal of Developmental Psychology 9(2). 257-270.

Yuill, Nicola \& Sarah Little. 2018. Thinking or feeling? An exploratory study of maternal scaffolding, child mental state talk, and emotion understanding in language-impaired and typically developing school-aged children. British Journal of Educational Psychology 88(2). 261-283.

\title{
Bionotes
}

\author{
Ilaria Gabbatore \\ University of Turin, Turin, Italy \\ ilaria.gabbatore@unito.it
}

Ilaria Gabbatore, PhD is a psychologist and currently a postdoctoral researcher at the Department of Psychology, University of Turin (Italy). She got her PhD in Neuroscience - Cognitive Science at the University of Turin. Later, she has worked at the Research Unit of Logopedics, University of Oulu, Finland. Her research interests lie in the field of pragmatic communication, from both a developmental and a neuropsychological perspective, with a particular interest on the crosscultural aspects of pragmatic development.

\section{Francesca M. Bosco}

University of Turin, Turin, Italy

Francesca M. Bosco, PhD is a cognitive psychologist and cognitive psychotherapist, full professor in General psychology and Psychology of communication and communication disorders at the Department of Psychology, University of Turin, Italy. She is coordinator of the GIPSI - research Group on Inferential Processes in Social Interaction at the University of Turin. Her research interests concern the cognitive processes underlying pragmatic communication in healthy and pathological conditions, with a particular attention to developmental aspects and to the rehabilitation of communicative abilities.

\section{Leena Mäkinen}

University of Oulu, Oulu, Finland

Leena Mäkinen, $\mathrm{PhD}$ is a researcher and speech-language pathologist at the Research Unit of Logopedics in the University of Oulu, Finland. Her research interests are in children's language development and its disorders. In particular, she has focused on narrative skills in typical development, Specific Language Impairments and Autism Spectrum Disorder.

\section{Eeva Leinonen}

Murdoch University, Perth, Australia

Eeva Leinonen is Vice Chancellor at Murdoch University. Professor Leinonen has an academic background in linguistics and psychology and has extensive experience in higher education in the United Kingdom, Europe and internationally. Her academic research focuses on pragmatic deficits 
of children with autistic spectrum disorders as well as higher education topics relating to curriculum and use of technology in learning.

\section{Soile Loukusa}

University of Oulu, Oulu, Finland

Soile Loukusa, PhD Speech Language Therapist, is adjunct professor at the Research Unit of Logopedics in the University of Oulu, Finland. Her research focuses on pragmatic communication abilities and social cognition skills in typical development as well as in individuals with autism spectrum disorder, developmental language disorder and attention deficit hyperactivity disorder. 